\title{
Independent Prognostic Value of Serum Markers in Diffuse Large B-Cell Lymphoma in the Era of the NCCN-IPI
}

\author{
Thomas Melchardt, $\mathrm{MD}^{\mathrm{a},{ }^{*}}$; Katharina Troppan, $\mathrm{MD}^{\mathrm{b}, *}$; Lukas Weiss, MDa ; Clemens Hufnagl, MSc ${ }^{\mathrm{a}}$; \\ Daniel Neureiter, MD; Wolfgang Tränkenschuh, MDc; Konstantin Schlick, MDa; Florian Huemer, MD; \\ Alexander Deutsch, MD ${ }^{\mathrm{b}}$; Peter Neumeister, $\mathrm{MD}^{\mathrm{b}}$; Richard Greil, MDª ${ }^{\mathrm{a}}$ Martin Pichler, MD ${ }^{\mathrm{d}}$; and \\ Alexander Egle, $\mathrm{MD}^{\mathrm{a}}$
}

\begin{abstract}
Background: Several serum parameters have been evaluated for adding prognostic value to clinical scoring systems in diffuse large B-cell lymphoma (DLBCL), but none of the reports used multivariate testing of more than one parameter at a time. The goal of this study was to validate widely available serum parameters for their independent prognostic impact in the era of the National Comprehensive Cancer Network-International Prognostic Index (NCCN-IPI) score to determine which were the most useful. Patients and Methods: This retrospective bicenter analysis includes 515 unselected patients with DLBCL who were treated with rituximab and anthracycline-based chemoimmunotherapy between 2004 and January 2014. Results: Anemia, high C-reactive protein, and high bilirubin levels had an independent prognostic value for survival in multivariate analyses in addition to the NCCN-IPI, whereas neutrophil-to-lymphocyte ratio, high gammaglutamyl transferase levels, and platelets-to-lymphocyte ratio did not. Conclusions: In our cohort, we describe the most promising markers to improve the NCCN-IPI. Anemia and high C-reactive protein levels retain their power in multivariate testing even in the era of the NCCNIPI. The negative role of high bilirubin levels may be associated as a marker of liver function. Further studies are warranted to incorporate these markers into prognostic models and define their role opposite novel molecular markers. (J Natl Compr Canc Netw 2015;13:1501-1508)
\end{abstract}

\section{Background}

Diffuse large B-cell lymphoma (DLBCL) is the most common histologic subtype of all non-Hodgkin's lymphomas (NHLs) and comprises approximately 20\% of newly diagnosed lymphoid neoplasms. ${ }^{1}$ Although an increasing knowledge of biology in DLBCL has led to the proposal of molecular tools for risk stratification, such as cell of origin (COO) assignment or analysis of Myc or $\mathrm{Bcl} 2$ status, relevant problems in the definitions of technical aspects in both $\mathrm{COO}$ and "double-hit lymphoma" definitions have led to a delay in translating this knowl-

From the aDepartment of Internal Medicine III, Cancer Research Institute, Paracelsus Medical University Salzburg, Salzburg, Austria; 'bivision of Hematology, Medical University of Graz, Graz, Austria; Institute of Pathology, Paracelsus Medical University Salzburg, Salzburg, Austria; and dDepartment of Experimental Therapeutics, The University of Texas MD Anderson Cancer Center, Duarte, Texas.

*These authors contributed equally to this manuscript.

The authors have disclosed that they have no financial interests, arrangements, affiliations, or commercial interests with the manufacturers edge into clinical cohorts outside of clinical trials. Thus, most risk assessments in everyday clinical practice are still performed using clinically available parameters. To this end, an enhanced National Comprehensive Cancer Network-International Prognostic Index (NCCNIPI) was recently proposed in patients treated with $\mathrm{R}$ CHOP (rituximab, cyclophosphamide, doxorubicin, vincristine, and prednisone) in North America. The NCCN-IPI incorporates the impact of very high lactate dehydrogenase levels as the only laboratory value in the list of parameters, in addition to very old age, and outperforms the traditional IPI., ${ }^{2,3}$

of any products discussed in this article or their competitors. This work was supported by the Paracelsus Medical University (PMU Grant: E13/17/089MEG).

T.M, K.T., and A.E were primarily responsible for the drafting of manuscript. All authors were involved in the management of the patients, and wrote and critically revised the manuscript.

Correspondence: Alexander Egle, MD, Department of Internal Medicine III, Salzburg Cancer Research Institute, Paracelsus Medical University, MüllnerHauptstrasse 48 - 5020, Salzburg, Austria. E-mail: a.egle@salk.at 
We recently validated this superior risk score in a European cohort. ${ }^{4}$ In the past, a number of laboratory markers have been proposed for prognostication in DLBCL. We have previously shown the additional independent value of serum albumin and $\beta 2$ microglobulin, ${ }^{4}$ but both parameters are not routinely available in large registries of patients with DLBCL.

We thus set out to define which more commonly available laboratory parameters may be suitable to help improve the prognostication in DLBCL. Markers such as C-reactive protein (CRP), lymphocytes, and neutrophils are available in most patients with DLBCL in clinical practice. These markers of inflammation are among the most frequently reported and published laboratory parameters in prognostication for malignant diseases. Persistent inflammation with increased acute phase protein, such as CRP or granulocytes, is a well-known cause of anemia, and overall is associated with a worse prognosis in lymphoma and other malignancies. ${ }^{5-10}$ In addition, liver function tests are generally available at baseline, but although lymphoma treatment efficiency or toxicity may be relatively determined by liver performance, no analyses on DLBCL prognosis have been reported.

Importantly, the best choice of laboratory markers in clinical practice was not clear in the past, because such markers were never included in multivariate analysis alongside each other.

The goal of this work was to assess the independent prognostic role of these serum parameters, and to determine a possible correlation between them in the era of the NCCN-IPI. Markers with an independent and reproducible influence on the clinical outcome may further improve the NCCN-IPI.

\section{Patients and Methods}

This retrospective analysis was approved by the Ethics Committee of the provincial government of Salzburg, Austria (415-EP/73/127-2012), and the local ethical committee of the Medical University of Graz (No. 25-434 ex 12/13). All patients included for this analysis were diagnosed with de novo DLBCL between 2004 and January 2014. Patients were HIVnegative and treated with $\mathrm{R}-\mathrm{CHOP}$ or R-CHOPlike chemoimmunotherapy as first-line treatment at the Third Medical Department of the Paracelsus Medical University Salzburg, Austria, and the Division of Hematology of the Medical University of
Graz, Austria. Clinical data, including the ECOG performance status, B symptoms, stage according to the Ann Arbor staging system, overall survival (OS), and progression-free survival (PFS), were retrospectively analyzed through chart-based review. All included laboratory parameters were routinely assessed prior to the start of chemotherapy. For patients who did not attend follow-up visits, clinical follow-up data were obtained via telephone interviews with the patients' general practitioner. All statistical analyses were performed using IBM SPSS statistics software, version 21. Mann-Whitney U test, Spearman rank order correlation, and Pearson chi-square test were used for univariate analyses, where appropriate. Survival was estimated using Kaplan-Meier curve analysis, with statistical comparison using the log-rank statistic. A 2-tailed significance level of 0.05 was considered statistically significant. Only statistically significant factors were included in multivariate Cox-regression analyses. Cutoff values in our cohort were determined using the receiver operating characteristic (ROC) calculation and Youden index analysis for OS.

\section{Results}

\section{Patient Characteristics}

We included 515 patients consecutively diagnosed with DLBCL treated between 2004 and January 2014 in this retrospective analysis (Table 1). In detail, the mean age of the whole cohort was 65.3 years (range, 20-92 years) and $52.4 \%$ of the patients were male. Ann Arbor stage III and IV were present in 47.6\%, and nonpegylated liposomal doxorubicin (NPLD) was used in $26 \%$ of the cases. The median follow-up for all patients alive was 53 months.

\section{Prognostic Impact of the NCCN-IPI}

Applying the NCCN-IPI in our patient cohort resulted in $9.3 \%$ of patients classified as low risk, $39.8 \%$ as lowintermediate, $37.3 \%$ as high-intermediate, and $13.2 \%$ as high risk (Table 1). As expected, the NCCN-IPI identified 4 prognostic groups of patients with highly different clinical outcomes (3-year PFS: 88.9\%, 73.8\%, $62.8 \%, 38.9$, respectively; $P<.001$, and 3 -year OS: $97.7 \%, 84.4 \%, 66.8 \%, 41.0 \%$, respectively; $P<.001)$.

\section{Serum Markers in DLBCL}

Several serum markers have been separately analyzed for their influence on the prognosis of patients with 


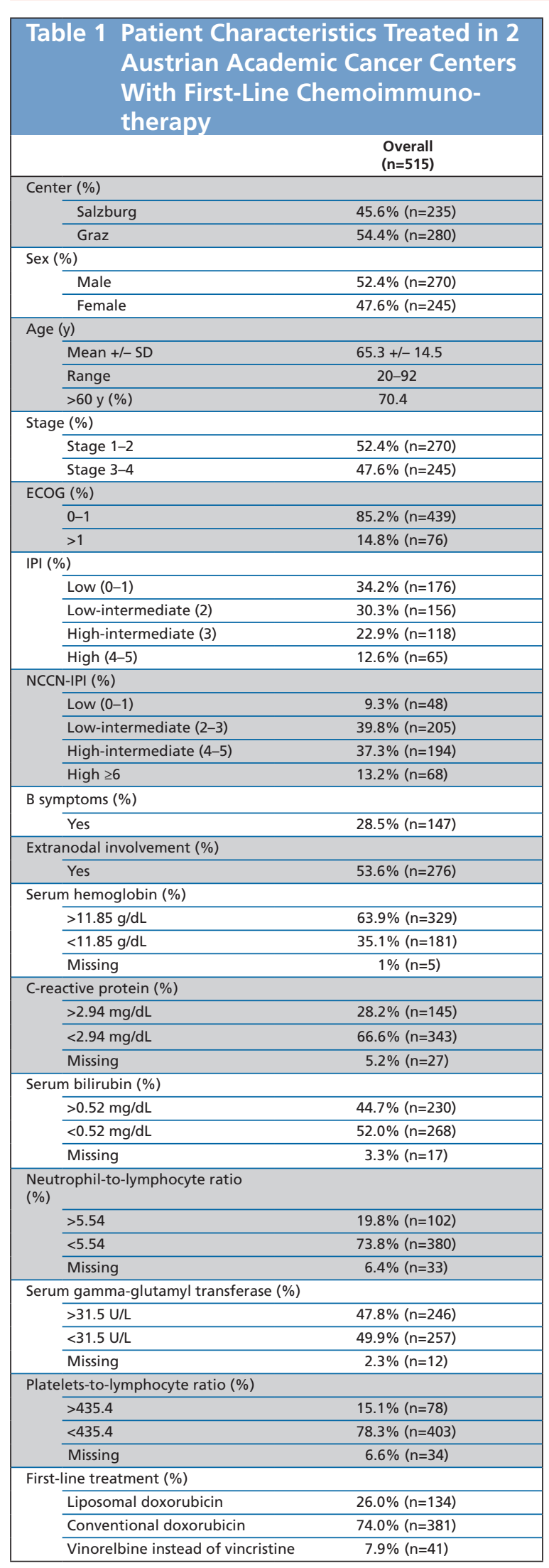

DLBCL and possible improvement of the IPI and NCCN-IPI in the past. Nevertheless, multivariate testing for an independent prognostic impact of these markers has not been reported so far. To determine the components for our multivariate analysis, available serum markers were first analyzed in univariate fashion.

\section{Markers of Inflammation and Their Influence on OS}

We and others ${ }^{10-12}$ have already shown the negative prognostic value of anemia in patients with lymphoma. In our cohort, an interpretable hemoglobin level before treatment was available in 510 patients (99\%; Table 1). A cutoff value of 11.85 $\mathrm{g} / \mathrm{dL}$ was determined to be optimal to discriminate the OS. Median PFS and OS were significantly lower in patients with low hemoglobin levels compared with patients with higher values (median PFS, 42 months vs not reached; $P<.001$; median OS, 55 months vs not reached; $P<.001$; Figure $1 \mathrm{~A}$ ).

High levels of CRP, an acute phase protein, are associated with worse clinical outcome in lymphoid and solid malignancies. ${ }^{7,8,13,14}$ Pretherapeutic CRP levels were available in 488 patients (94.8\%; Table 1). A cutoff value of $2.94 \mathrm{mg} / \mathrm{dL}$ was determined by ROC and Youden analysis for discrimination of OS. Higher CRP levels were significantly associated with worse PFS and OS (median PFS, 23 months vs not reached; $P<.001$; median OS, 50 months vs not reached; $P<.001$; Figure 1B).

The negative effect of a high neutrophil-tolymphocyte ratio (NLR) was previously reported in smaller single-center cohorts. ${ }^{6,15}$ Pretherapeutic NLR was available in 482 patients $(93.6 \%$; Table 1 ) and a cutoff value of 5.54 was found to be optimal to discriminate the OS. A higher ratio was associated with worse PFS (median PFS, 64 months vs not reached; $P=.017$ ) and worse OS (median OS, 65 months vs not reached; $P=.004$ ).

Platelets-to-lymphocyte ratio (PLR) is reported to be a prognostic factor in several solid malignancies, ${ }^{16-21}$ but no data yet exist about patients with lymphoma. PLR was available in 481 patients (93.4\%; Table 1). A cutoff value of 435.4 was determined by ROC and Youden analysis to be optimal to discriminate OS. Nevertheless, this cutoff had no significant effect on PFS (median PFS, 91 vs 96 months; $P=.115$ ) and OS (median OS, 115 months vs not reached; $P=.096$ ).

\section{Liver Serum Markers in DLCBL}

Despite its wide availability during routine assessment, there are only small reports about the role 

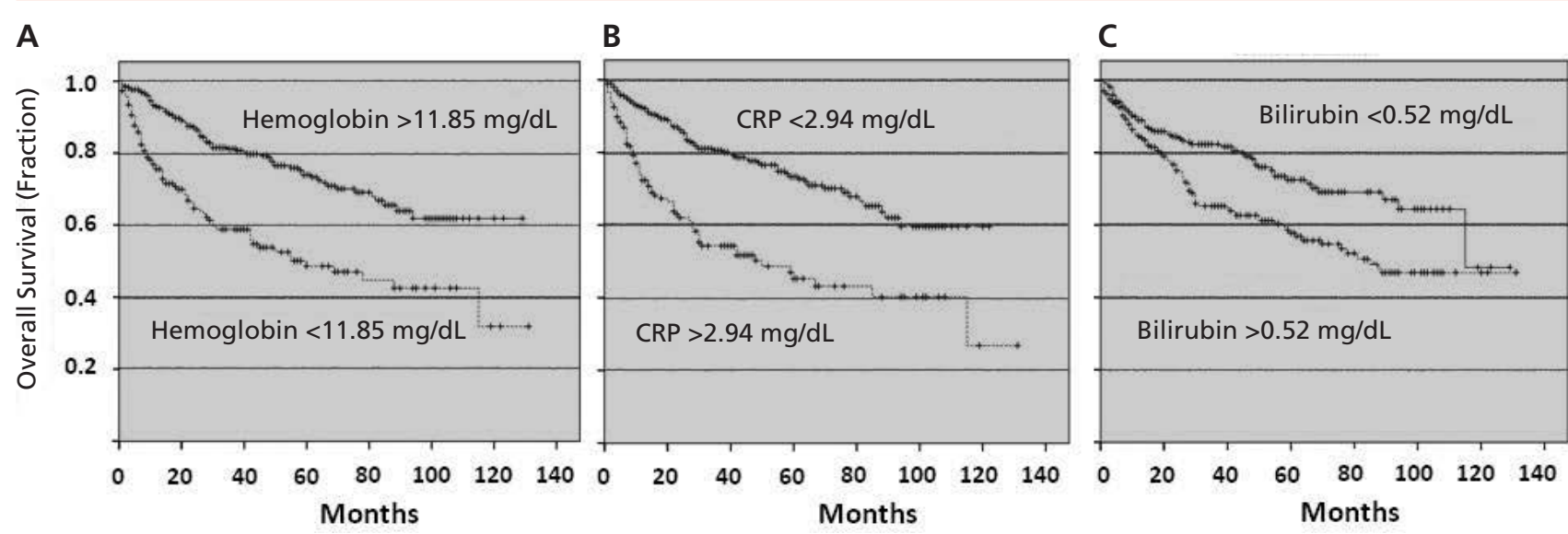

Figure 1 Outcome according to serum parameters assessed during clinical routine. (A) Anemia with a cutoff value of $11.85 \mathrm{mg} / \mathrm{dL}$ was associated with a lower overall survival ([OS] median OS: 55 months vs not reached; $P<.001$ ). (B) High C-reactive protein (CRP) levels with a cutoff value of 2.94 $\mathrm{mg} / \mathrm{dL}$ were associated with a lower OS (median OS: 50 months vs not reached; $P<.001$ ). (C) High bilirubin levels with a cutoff value of $0.52 \mathrm{mg} / \mathrm{dL}$ were associated with a lower OS (median OS: 85 vs 115 months; $P=.001$ ).

of hyperbilirubinemia in patients with hematologic malignancies. ${ }^{22-25}$ Serum bilirubin levels were available in $96.7 \%$ of our patients $(n=498$; Table 1$)$. A cutoff value of $0.52 \mathrm{mg} / \mathrm{dL}$ was found to be optimal by ROC analysis to discriminate the OS. Higher values were associated with a worse OS (median OS, 85 vs 115 months; $P=.001$; Figure $1 C$ ), and a trend for worse PFS that did not reach statistical significance (median PFS, 65 vs 115 months; $P=.078$ ).

Serum levels of the gamma-glutamyl transferase (GGT) were available in 503 patients (97.7\%; Table 1). A cutoff value of $31.5 \mathrm{U} / \mathrm{L}$ was determined to be optimal to discriminate the OS. Higher GGT levels were associated with a worse PFS (median PFS, 65 vs 115 months; $P=.018$ ) and worse OS (median OS, 88 vs 115 months; $P=.011$ ).

\section{Independent Value of Serum Markers in Patients Assessed With the NCCN-IPI}

Upon analyzing already described inflammation markers, we detected statistically significant, but at best moderate correlations, between CRP and hemoglobin levels (Spearman's correlation coefficient $\left[r_{s}\right] ; 0.472$; $P<.0001)$, CRP and NLR ( $r, 0.32 ; P<.0001)$, and NLR and hemoglobin levels $\left(r_{s}, 0.207 ; P<.0001\right)$. Correlation between GGT and bilirubin serum levels was also weak but statistically significant $\left(r_{s}, 0.118 ; P<.009\right)$.

To elucidate the additional value of the described parameters in the era of the NCCN-IPI, we performed a multivariate analysis in 450 patients with all tested parameters available on OS. Multivariate testing of all significant parameters in univariate testing revealed an independent prognostic role of hemoglobin, CRP, and bilirubin in addition to the NCCN-IPI. PLR failed to show any prognostic information in the preceding univariate testing, and GGT levels and NLR failed to show correlation with OS (Table 2) in multivariate testing.

We also analyzed the influence of these markers on PFS. High bilirubin level and PLR had no influence on PFS in univariate testing, and hemoglobin and CRP levels retained their prognostic impact on PFS compared with GGT level and NLR in multivariate testing in addition to the NCCN-IPI.

We also tested the value of anemia and increased CRP levels to refine the prognostic accuracy of the NCCN-IPI. Both variables could not significantly refine the prognosis of patients with very good prognosis according to the NCCN-IPI (low and lowintermediate risk groups) or of patients with poor survival (high-risk group). Nevertheless, within the high-intermediate risk group, the presence of none, one, or both variables could determine more precisely the prognosis (median PFS and OS: 94 months and not reached, respectively, for no anemia and no increased CRP vs 55 and 67 months, respectively, for anemia or increased CRP vs 16 and 23 months, respectively, for anemia and increased CRP; $P<.001$ for both; Figure 2).

\section{Discussion}

Risk prognostication in patients with DLBCL has been based on clinical parameters for more than 20 years. This practice has been challenged by prognostic tools based on new molecular insights in the 
Routine Serum Markers in DLBCL

\begin{tabular}{|c|c|c|c|c|c|c|c|c|}
\hline & \multicolumn{4}{|c|}{ Univariate } & \multicolumn{4}{|c|}{ Multivariate } \\
\hline & HR & $95 \% \mathrm{Cl}$ & $P$ Value & $\mathbf{n}$ & HR & $95 \% \mathrm{Cl}$ & $P$ Value & n \\
\hline \multicolumn{9}{|l|}{ Sex } \\
\hline Female vs male & 0.828 & $0.603-1.136$ & .242 & 515 & NA & & & \\
\hline \multicolumn{9}{|l|}{ NCCN-IPI } \\
\hline Low vs high & 3.180 & $2.237-4.521$ & $<.001$ & 515 & 2.063 & $1.379-3.088$ & $<.001$ & 450 \\
\hline \multicolumn{9}{|l|}{ Hemoglobin } \\
\hline$>11.85$ vs $<11.85 \mathrm{~g} / \mathrm{dL}$ & 2.415 & $1.759-3.316$ & $<.001$ & 510 & 1.577 & $1.082-2.299$ & .018 & 450 \\
\hline \multicolumn{9}{|l|}{ C-reactive protein } \\
\hline$>2.94$ vs $<2.94 \mathrm{mg} / \mathrm{dL}$ & 2.518 & $1.817-3.488$ & $<.001$ & 488 & 1.679 & $1.137-2.453$ & .009 & 450 \\
\hline \multicolumn{9}{|l|}{ Bilirubin } \\
\hline$>0.52$ vs $<0.52 \mathrm{mg} / \mathrm{dL}$ & 1.719 & $1.239-2.385$ & .001 & 498 & 1.517 & $1.066-2.159$ & .021 & 450 \\
\hline \multicolumn{9}{|l|}{ Neutrophil-to-lymphocyte ratio } \\
\hline$>5.54$ vs $<5.54$ & 1.623 & $1.167-2.258$ & .004 & 482 & 1.141 & $0.796-1.635$ & .474 & 450 \\
\hline \multicolumn{9}{|l|}{ Gamma-glutamyl transferase } \\
\hline$>31.5$ vs $<31.5 \mathrm{U} / \mathrm{L}$ & 1.512 & $1.097-2.084$ & .011 & 503 & 1.247 & $0.873-1.782$ & .224 & 450 \\
\hline \multicolumn{9}{|l|}{ Platelets-to-lymphocyte ratio } \\
\hline$>435.4$ vs $<435.4$ & 1.387 & $0.940-2.048$ & .099 & 481 & NA & & & \\
\hline
\end{tabular}

Abbreviations: HR, hazard ratio; IPI, International Prognostic Index; NA, not applicable; NCCN, National Comprehensive Cancer Network.

pathobiology of DLBCL. ${ }^{26-29}$ Because of problems in standardization or reproducibility of tests or the lack of universally accepted definitions of risk, or as a result of simple lack of availability, these tools, such as $\mathrm{COO}$ or double-hit lymphoma assessment, are not regularly incorporated into clinical practice in most parts of the world, and their influence on treatment stratification is unclear.

Various algorithms of immunohistochemical staining used for $\mathrm{COO}$ analyses have been proposed, but their concordance with functional microarray analyses and their influence on clinical outcome differs in the literature. ${ }^{30-32}$ Despite combined assessment of microarray and immunohistochemical data the $\mathrm{COO}$ definition added no additional prognostic value to the IPI in the largest cohort study published so far. ${ }^{33}$ New techniques such as the nanostring technology may enhance reproducibility and efficacy of these analyses, as suggested in a cohort published by the British Columbia Cancer Agency. This approach added further information to the IPI assessment with the latter still remaining the most powerful prognostication tool, but no significant clinical influence of the immunohistochemical assessment of c-Myc and $\mathrm{Bcl}-2$ was found in multivariate analyses. ${ }^{34}$
Because of these limitations, there is a relevant clinical need for a more accurate prognostication in patients with DLBCL. The recently established NCCN-IPI risk score, including no molecular information, has been shown to be superior to the conventional $\mathrm{IPI}^{2,4}$ and may be implemented as novel standard risk prediction tool in patients with newly diagnosed DLBCL. However, although high and low risk are assessed very accurately, there remains a large intermediate group with statistically highly significant fates but a low power to predict the outcome of an individual, limiting the usable information for most patients. An improvement in this group would be worthwhile. We and others have investigated the role in risk prediction of serum markers of chronic inflammation in addition to the IPI in the past, but one major limitation of these reports was the missing multivariate testing within these markers.

We assessed an array of such parameters for their independent impact on prognosis in multivariate analyses in a large bicenter analysis of 515 patients. We could confirm the previously reported prognostic impact of elevated CRP and anemia. ${ }^{7,10,35}$ Despite a weak correlation, they were significantly associated with a shorter OS and proved to have independent 
power from each other in multivariate analysis. Both parameters may indicate inflammation caused by the lymphoma or individual comorbidity, and the tumor-promoting effect of inflammatory cytokines has already been described. As an example, high IL-6 serum levels, a major factor for the development of anemia in lymphoma, have been described to be associated with poor outcome in patients with DLBCL. ${ }^{36}$ Furthermore, our results suggest a relatively smaller prognostic role of the NLR in DLCBL in contrast to findings of previous reports when analyzed in multivariate testing. ${ }^{6,15}$

As stated earlier, the NCCN-IPI provides very clear prognostic information in high- and low-risk situations, but the relative large intermediate-high risk group, especially, follows a course that is still highly variable. As judged from our cohort, elevated CRP and anemia may be used to refine the prognosis of patients within this high-intermediate NCCNIPI group. This patient group has an intermediate prognosis (median PFS and OS: 65 and 85 months, respectively) as a whole, but adding these variables separated 3 groups of patients with highly different PFS and OS. Patients with anemia and increased CRP are lined up with the high-risk NCCN-IPI group, and those with normal hemoglobin and low CRP followed a similar course to that of the lowintermediate group. This may be of clinical impor-

A

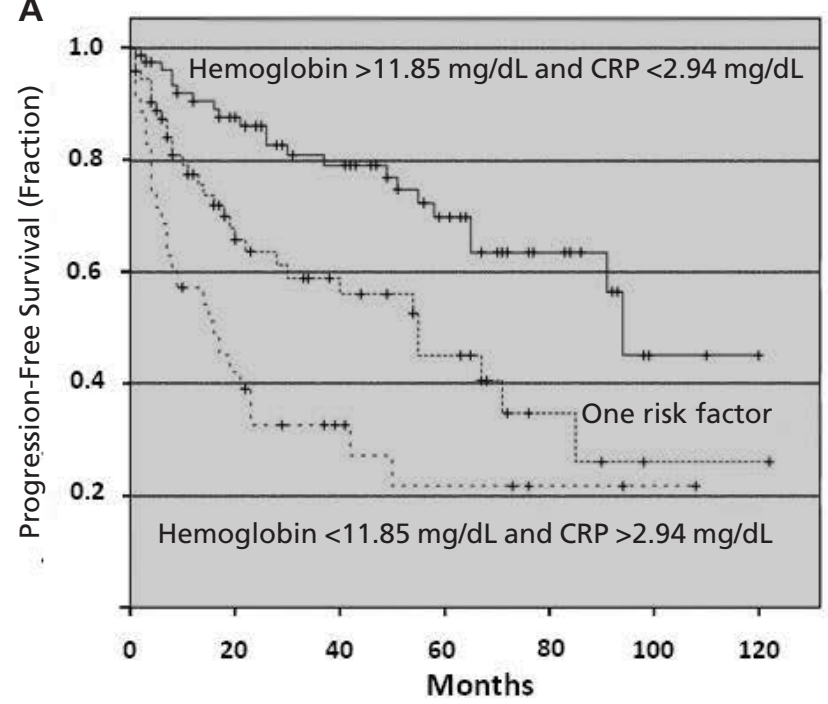

tance, because this group with an intermediate risk may include the most patients, who may benefit from more intensive or novel therapy approaches.

We also assessed liver function parameters for their prognostic power, and found a negative prognostic role of higher bilirubin levels in patients with DLBCL treated with R-CHOP. This effect may seem expected in patients with high levels of bilirubin caused by overt liver damage, but we detected a highly significant cutoff value of 0.52 $\mathrm{mg} / \mathrm{dL}$, which is still in the normal range of the average population. When we tested the prognostic impact of the upper level of the normal range, 1.2 $\mathrm{mg} / \mathrm{dL}$, this cutoff lost its independent influence on OS during multivariate analyses. Additionally, when excluding all patients with bilirubinemia associated with local infiltration of DLBCL and all those with bilirubin higher than $2.0 \mathrm{mg} / \mathrm{dL}$, both significance and cutoff held up, suggesting that a variation within the normal range of bilirubin was responsible for our finding. A similar phenomenon was observed regarding GGT in univariate testing. It is thus possible that variations in normal liver function may result in different efficacy of the glucuronidation system, determining the outcomes of chemoimmunotherapy in DLBCL. Furthermore, because of a significant association of higher bilirubin levels with OS, but not PFS, a role as a sur-

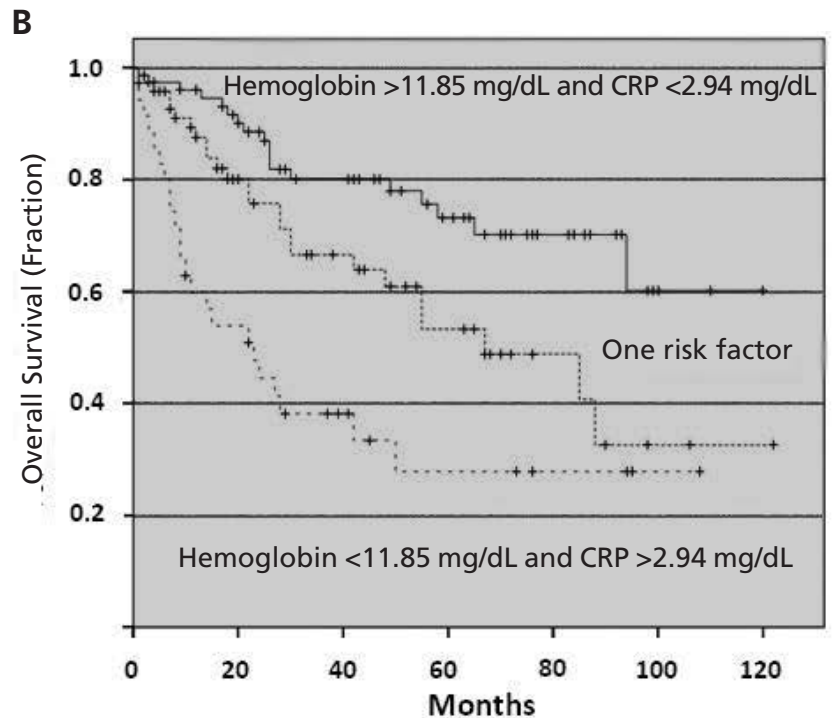

Figure 2 Outcome in patients with high-intermediate National Comprehensive Cancer Network-International Prognostic Index (NCCN-IPI). Anemia and increased C-reactive protein (CRP) levels improved the prognostic accuracy within the high-intermediate NCCN-IPI group. The presence of none, one, or both variables could state more precisely the progression-free survival (PFS; Figure 2A) and overall survival (OS; Figure 2B): median PFS and OS: 94 months and not reached, respectively, for no anemia and no increased CRP vs 55 and 67 months, respectively, for anemia or increased CRP vs 16 and 23 months, respectively, for anemia and increased CRP; $P<.001$ for both. 
rogate parameter of general health and comorbidity instead of tumor control is also possible. Although we can only speculate about this, our work may inspire further analyses in this setting. A better understanding of these parameters is needed, because it would clearly be difficult to counsel patients regarding their risk using variations of normal laboratory values. Our data on liver function tests are thus hypothesis-generating, rather than easily clinically applicable. Overall, our results confirm the importance of multivariate testing during evaluation of the additional prognostic value of new biomarkers.

Our report is not without limitations, because retrospective analyses are always prone to bias factors. Nevertheless, we consider a possible selection bias as relatively small because both participating cancer centers are responsible for the care of almost all patients with lymphoma in their districts. In addition, lymphoma treatment is not offered in smaller hospitals in their region and there are no hematologists and oncologists in private practice in Austria. Therefore, we think that the described cohort is relatively close to patients with DLBCL found in the "real-life setting."

It will be very important to validate such parameters opposite molecularly defined risk factors, and future cohorts will possibly need to include clinical and laboratory values and molecular markers in their multivariate models.

\section{Conclusions}

In this study, we show that CRP, hemoglobin, and bilirubin levels have an independent prognostic effect in patients with DLBCL treated with R-CHOP. Anemia and increased CRP levels seem to be the most promising markers to improve the prognostic accuracy of the NCCN-IPI, especially in patients with intermediate risk. Further efforts may show whether the incorporation of these markers may be able to further improve clinical prognostication.

\section{References}

1. Morton LM, Wang SS, Devesa SS, et al. Lymphoma incidence patterns by WHO subtype in the United States, 1992-2001. Blood 2006;107:265-276.

2. Zhou Z, Sehn LH, Rademaker AW, et al. An enhanced International Prognostic Index (NCCN-IPI) for patients with diffuse large B-cell lymphoma treated in the rituximab era. Blood 2014;123:837-842.

3. Ziepert M, Hasenclever D, Kuhnt E, et al. Standard International prognostic index remains a valid predictor of outcome for patients with aggressive CD20+ B-cell lymphoma in the rituximab era. J Clin Oncol 2010;28:2373-2380.
4. Melchardt T, Troppan K, Weiss L, et al. A modified scoring of the NCCNIPI is more accurate in the elderly and is improved by albumin and beta2 -microglobulin. Br J Haematol 2015;168:239-245.

5. Eatrides J, Thompson Z, Lee JH, et al. Serum albumin as a stable predictor of prognosis during initial treatment in patients with diffuse large B cell lymphoma. Ann Hematol 2015;94:357-358.

6. Troppan K, Deutsch A, Gerger A, et al. The derived neutrophil to lymphocyte ratio is an independent prognostic factor in patients with diffuse large B-cell lymphoma. Br J Cancer 2014;110:369-374.

7. Troppan KT, Schlick K, Deutsch A, et al. C-reactive protein level is a prognostic indicator for survival and improves the predictive ability of the R-IPI score in diffuse large B-cell lymphoma patients. Br J Cancer 2014;111:55-60.

8. Yi JH, Wang D, Li ZY, et al. C-reactive protein as a prognostic factor for human osteosarcoma: a meta-analysis and literature review. PLoS One 2014;9:e94632.

9. Talaulikar D, Choudhury A, Shadbolt B, Brown M. Lymphocytopenia as a prognostic marker for diffuse large B cell lymphomas. Leuk Lymphoma 2008;49:959-964.

10. Troppan KT, Melchardt T, Deutsch A, et al. The significance of pre-treatment anemia in the era of R-IPI and NCCN-IPI prognostic risk assessment tools: a dual-center study in diffuse large B-cell lymphoma patients [published online ahead of print February 13, 2015]. Eur J Haematol. doi: 10.1111/ejh.12529.

11. Moullet I, Salles G, Ketterer N, et al. Frequency and significance of anemia in non-Hodgkin's lymphoma patients. Ann Oncol 1998;9:1109-1115.

12. Boll B, Goergen H, Arndt N, et al. Relapsed hodgkin lymphoma in older patients: a comprehensive analysis from the German hodgkin study group. J patients: a comprehensive analy
Clin Oncol 2013;31:4431-4437.

13. Pathak S, Nunes QM, Daniels IR, Smart NJ. Is CRP useful in prognostication for colorectal cancer? A systematic review. Colorectal Dis 2014;16:769-776.

14. Dai J, Tang K, Xiao W, et al. Prognostic significance of $C$-reactive protein in urological cancers: a systematic review and meta-analysis. Asian Pac J Cancer Prev 2014;15:3369-3375.

15. Porrata LF, Ristow $K$, Habermann $T$, et al. Predicting survival for diffuse large B-cell lymphoma patients using baseline neutrophil/lymphocyte ratio. Am J Hematol 2010;85:896-899.

16. Smith RA, Bosonnet L, Raraty M, et al. Preoperative platelet-lymphocyte ratio is an independent significant prognostic marker in resected pancreatic ductal adenocarcinoma. Am J Surg 2009;197:466-472.

17. Smith RA, Ghaneh P, Sutton R, et al. Prognosis of resected ampullary adenocarcinoma by preoperative serum CA19-9 levels and platelet-lymphocyte ratio. J Gastrointest Surg 2008;12:1422-1428.

18. Smith RA, Bosonnet L, Ghaneh $\mathrm{P}$, et al. The platelet-lymphocyte ratio improves the predictive value of serum CA19-9 levels in determining patient selection for staging laparoscopy in suspected periampullary cancer. Surgery 2008;143:658-666.

19. Kwon HC, Kim SH, Oh SY, et al. Clinical significance of preoperative neutrophil-lymphocyte versus platelet-lymphocyte ratio in patients with operable colorectal cancer. Biomarkers 2012;17:216-222.

20. Raungkaewmanee $S$, Tangjitgamol $S$, Manusirivithaya $S$, et al. Platelet to lymphocyte ratio as a prognostic factor for epithelial ovarian cancer. J Gynecol Oncol 2012;23:265-273.

21. Lee S, Oh SY, Kim SH, et al. Prognostic significance of neutrophil lymphocyte ratio and platelet lymphocyte ratio in advanced gastric cancer patients treated with FOLFOX chemotherapy. BMC Cancer 2013;13:350.

22. Segal I, Rassekh SR, Bond MC, et al. Abnormal liver transaminases and conjugated hyperbilirubinemia at presentation of acute lymphoblastic leukemia. Pediatr Blood Cancer 2010;55:434-439.

23. Ross WA, Egwim CI, Wallace MJ, et al. Outcomes in lymphoma patients with obstructive jaundice: a cancer center experience. Dig Dis Sci 2010;55:32713277 .

24. Ghosh I, Bakhshi S. Jaundice as a presenting manifestation of pediatric nonHodgkin lymphoma: etiology, management, and outcome. J Pediatr Hematol Oncol 2010;32:e131-135.

25. Sharma PB, Karki L. Abnormal hepatic function and splenomegaly on the newly diagnosed acute leukemia patients. JNMA J Nepal Med Assoc 2007;46:165-169.

26. Alizadeh AA, Eisen MB, Davis RE, et al. Distinct types of diffuse large B-cell lymphoma identified by gene expression profiling. Nature 2000;403:503-511.

27. Barrans S, Crouch S, Smith A, et al. Rearrangement of MYC is associated with poor prognosis in patients with diffuse large B-cell lymphoma treated in the era of rituximab. J Clin Oncol 2010;28:3360-3365.

28. Ott G, Ziepert M, Klapper W, et al. Immunoblastic morphology but not the immunohistochemical GCB/nonGCB classifier predicts outcome in diffuse large B-cell lymphoma in the RICOVER-60 trial of the DSHNHL. Blood 2010;116:4916-4925.

29. Salles G, de Jong D, Xie W, et al. Prognostic significance of immunohistochemical biomarkers in diffuse large B-cell lymphoma: a study from the Lunenburg Lymphoma Biomarker Consortium. Blood 2011;117:7070-7078.

30. Choi WW, Weisenburger DD, Greiner TC, et al. A new immunostain algorithm classifies diffuse large B-cell lymphoma into molecular subtypes with high accuracy. Clin Cancer Res 2009;15:5494-5502.

31. Hans CP, Weisenburger DD, Greiner TC, et al. Confirmation of the molecular classification of diffuse large B-cell lymphoma by immunohistochemistry using a tissue microarray. Blood 2004;103:275-282. 
Melchardt et al

32. Meyer PN, Fu K, Greiner TC, et al. Immunohistochemical methods for predicting cell of origin and survival in patients with diffuse large B-cell lymphoma treated with rituximab. J Clin Oncol 2011;29:200-207.

33. $\mathrm{Hu} \mathrm{S}, \mathrm{Xu}-\mathrm{Monette} \mathrm{ZY}$, Tzankov A, et al. MYC/BCL2 protein coexpression contributes to the inferior survival of activated B-cell subtype of diffuse large B-cell lymphoma and demonstrates high-risk gene expression signatures: a report from The International DLBCL Rituximab-CHOP Consortium Program. Blood 2013;121:4021-4031.

34. Scott DW, Mottok A, Ennishi D, et al. Prognostic significance of diffuse large B-cell lymphoma cell of origin determined by digital gene expression in formalin-fixed paraffin-embedded tissue biopsies. J Clin Oncol 2015;33:28482856.

35. Cao Y, Shi YX, Chen JO, et al. Serum C-reactive protein as an important prognostic variable in patients with diffuse large B cell lymphoma. Tumour Biol 2012;33:1039-1044.

36. Giachelia M, Voso MT, Tisi MC, et al. Interleukin-6 plasma levels are modulated by a polymorphism in the NF-kappaB1 gene and are associated with outcome following rituximab-combined chemotherapy in diffuse large B-cell non-Hodgkin lymphoma. Leuk Lymphoma 2012;53:411-416. 